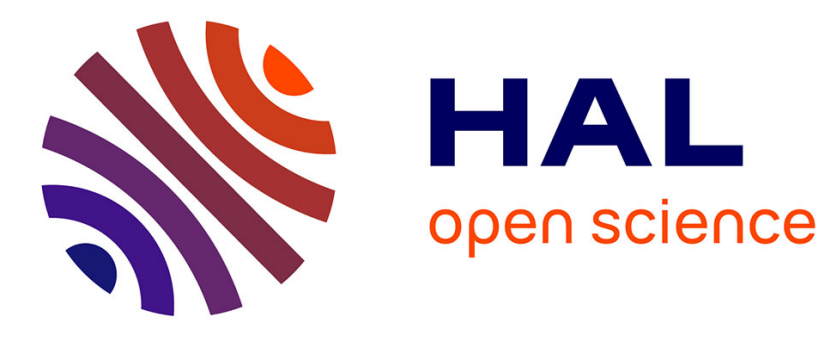

\title{
Integration of 3D Nanographene into Mesoporous Germanium
}

\author{
Stéphanie Sauze, Mohammad Reza Aziziyan, Pascal Brault, Gitanjali \\ Kolhatkar, Andreas Ruediger, Andreas Korinek, Denis Machon, Richard Arès, \\ Abderraouf Boucherif
}

\section{To cite this version:}

Stéphanie Sauze, Mohammad Reza Aziziyan, Pascal Brault, Gitanjali Kolhatkar, Andreas Ruediger, et al.. Integration of 3D Nanographene into Mesoporous Germanium. Nanoscale, 2020, 12, pp.23984.

10.1039/d0nr04937a . hal-02955987

\section{HAL Id: hal-02955987 \\ https://hal.science/hal-02955987}

Submitted on 2 Oct 2020

HAL is a multi-disciplinary open access archive for the deposit and dissemination of scientific research documents, whether they are published or not. The documents may come from teaching and research institutions in France or abroad, or from public or private research centers.
L'archive ouverte pluridisciplinaire HAL, est destinée au dépôt et à la diffusion de documents scientifiques de niveau recherche, publiés ou non, émanant des établissements d'enseignement et de recherche français ou étrangers, des laboratoires publics ou privés. 


\title{
Integration of 3D Nanographene into Mesoporous Germanium
}

Stéphanie Sauze, ${ }^{* \mathrm{a} b}$ Mohammad Reza Aziziyan, ${ }^{\mathrm{a} b}$ Pascal Brault, ${ }^{\mathrm{c}}$ Gitanjali Kolhatkar, ${ }^{\mathrm{d} \dagger}$ Andreas Ruediger, ${ }^{\mathrm{d}}$ Andreas Korinek, ${ }^{\mathrm{e}}$ Denis Machon, ${ }^{\mathrm{ab} f}$ Richard Arès ${ }^{\mathrm{a} b}$ and Abderraouf Boucherif*ab

a. Institut Interdisciplinaire d'Innovation Technologique (3IT), Université de Sherbrooke, 3000

Boulevard Université, Sherbrooke, J1K OA5 Québec, Canada

b. Laboratoire Nanotechnologies Nanosystèmes (LN2) - CNRS UMI-3463 Institut Interdisciplinaire d'Innovation Technologique (3IT), Université de Sherbrooke, 3000 Boulevard Université, Sherbrooke, J1K OA5 Québec, Canada.

c. GREMI, Groupe de Recherches sur l'Énergétique des Milieux Ionisés UMR7344, CNRS/Université d'Orléans, 14 rue d'Issoudun, BP 6744, 45067 Orléans Cedex 2, France

d. Nanoelectronics-Nanophotonics INRS-EMT, 1650, Boulevard Lionel-Boulet, Varennes, J3X 1S2

Québec, Canada

e. Canadian Centre for Electron Microscopy, McMaster University, 1280 Main Street West, Hamilton,

L8S 4L7, Ontario, Canada

f. Univ. Lyon, Université Claude Bernard Lyon 1, CNRS, Institut Lumière Matière, F-69622 Lyon, France

$\dagger$ Nanoelectronics, Faculty of Engineering, Kiel University, Kaiserstraße 2, 24143 Kiel, Germany

(Present address for Dr. Gitanjali Kolhatkar)

Corresponding authors email: stephanie.sauze@usherbrooke.ca,

a.boucherif@usherbrooke.ca

\begin{abstract}
Graphene is a key material of interest for modification of physicochemical surface properties. However, its flat surface is a limitation for applications requiring a high specific surface area. This restriction may be overcome by integrating 2D materials in a 3D structure. Here, a strategy for the controlled synthesis of Graphene-Mesoporous Germanium (Gr-MP-Ge) nanomaterials is presented. Bipolar electrochemical etching and chemical vapor infiltration were employed for, respectively, nanostructuration of Ge substrate and subsequent 3D nanographene coating. While Raman spectroscopy reveals tunable domain size of nanographene with the treatment temperature, transmission electron microscopy data confirm that the crystallinity of Gr-MP-Ge is preserved. X-ray photoelectron spectroscopy indicates the non-covalent bonding of carbon to Ge for Gr-MP-Ge. State-of-the-art molecular dynamics modeling allows for a deeper understanding of the synthesis process through the presence of radicals. The successful synthesis of this nanomaterials offers integration of nanographene into a 3D structure with a high aspect ratio and light weight, thereby opens avenues to a variety of applications for this versatile nanomaterial.
\end{abstract}




\section{Introduction}

Initially known as a fundamental object of study, graphene has evolved to become one of the most important research fields in materials science with a significant potential in a wide variety of applications., ${ }^{1,2}$ However, a major challenge for such applications is large-scale synthesis of graphene and its implementation in $3 \mathrm{D}$ systems. ${ }^{3}$ In fact, its $2 \mathrm{D}$-planar structure is a limitation preventing large-scale applications requiring high specific surface. It is then of the first interest to bring graphene into a 3D structure but not at the detrimental of the graphene quality and properties. Graphene has already been handled into different forms such as quantum $\operatorname{dots}^{4-7}$ and nanoribbons ${ }^{8}$ for use in semiconductor devices or else as foams ${ }^{9}$ and hydrogels ${ }^{10}$ for energy and biological applications. It is important for some applications to have a high specific surface area in a small volume (ratio surface/volume high) as for energy storage in portable electronics and drug delivery applications, and such a characteristic appeared to be the source of promising or enhanced performance. ${ }^{11-13}$ Nevertheless, the integration of graphene inside the 3D structure via a simple and direct method is challenging. In addition, nanostructured materials showed the emergence of new properties with the decreasing sizes, a catalytic surface for the graphene growth and combination with graphene offers hybrid properties. ${ }^{14,15}$

The strategy developed in this work proposes to use mesoporous semiconductor to increase the specific surface area with a 3D structure. For this purpose, we used germanium $(\mathrm{Ge})$, as it is one of the semiconductors that plays an important role in development of solid-state devices, mainly due to its specific characteristics that distinguish it from other group IV and III-V materials. ${ }^{16}$ Besides, it has attracted a lot of interest for direct growth of graphene. Primarily, Ge has catalytic activity allowing a decrease of the energy barrier for the decomposition of the carbon precursor. ${ }^{17}$ Moreover, carbon has a low solubility in Ge even at its melting temperature $\left(\approx 10^{8}\right.$ atoms $/ \mathrm{cm}^{3}$ at the melting point) and, lastly, there is a small difference between the thermal expansion coefficients of Ge and carbon. ${ }^{18,19}$ These characteristics drastically decrease the wrinkle formation in graphene layers when synthesized over a Ge surface. ${ }^{20}$ All these aspects make Ge a prominent candidate for the growth of graphene with a direct approach.

Before the direct growth of graphene, a nanostructuration step is necessary in order to increase the specific surface area of germanium. Electrochemical etching has already been used in industrial manufacturing and it is quite compatible with semiconductor manufacturing processes. Electrochemical etching or anodization has offered one of the leading platforms for well-controlled fabrication of porous silicon ${ }^{21}$ and this method has been extended to the Ge substrates using direct current. ${ }^{16,22}$ Further works led to development of a method based on bipolar pulses, known as 
Bipolar Electrochemical Etching (BEE), to induce pore formation and pore passivation with cycles of positive and negative current pulses. ${ }^{23}$ Recently, different electrochemical regimes have been developed for anodization of Ge and optimizing lateral homogeneity of the MP-Ge films. ${ }^{24-26}$ The preceding discussion points out that combination of mesoporous $\mathrm{Ge}(\mathrm{MP}-\mathrm{Ge})$ and graphene is an avenue to use the large specific area of graphene in a semiconductor, in order to design a versatile 3D nanostructured material. The targeted material consists of an MP-Ge substrate, with crystalline structure, coated with a thin layer of graphene encapsulating all the porous structure (See Figure 1). In this work, we demonstrate a new path for the synthesis of 3D nanographene within a MP-Ge substrate. Our strategy is a two-step process using bipolar electrochemical etching of germanium wafers followed by a Chemical Vapor Infiltration (CVI) of acetylene, at atmospheric pressure. On this subject, Chemical Vapor Deposition (CVD) was demonstrated in 2009 for large-area graphene growth synthesis, but some issues still remain unresolved. ${ }^{27-30}$ At the moment, CVD is considered as the most promising method for growth of single-crystal and large-area graphene with higher quality of the graphene compared with most other methods. ${ }^{18}$

Extensive characterizations have been performed to fully describe the resulting materials. First, the graphene coating was confirmed with Raman spectroscopy analyses. Then, the crystalline structure and chemical compositions of the produced nanomaterials were investigated using Transmission Electron Microscopy (TEM) and X-ray Photoelectron Spectroscopy (XPS) measurements. To further clarify the growth mechanism at different temperatures, molecular dynamics modeling was carried out.

We anticipate that the material we developed and optimized will be of great interest in diversified applications. For instance, tuning the crystalline quality of the graphene coating will allow for controlling associated properties such as electric and thermal conductivity. In addition, the nanocomposite is well adapted to the microelectronics industry as the synthesis is compatible with microfabrication line industry. This may be a way to introduce graphene in microelectronic devices.

\section{Experimental}

Synthesis of Graphene-Mesoporous Germanium (Gr-MP-Ge) nanomaterials was carried out through the following three-step process: 1. Anodization of the Ge wafer using Bipolar Electrochemical Etching (BEE) on bulk Ge wafers, 2. Acetylene infiltration and 3. Graphene formation within the porous structure through thermal treatment. The combination of the last two steps is identified as Chemical Vapor Infiltration (CVI). 
Bipolar Electrochemical Etching (BEE): For the anodization step, a one side polished p-type Gallium doped Ge wafer was used with the following characteristics: resistivity of 0.01-0.02 $\Omega . \mathrm{cm}$, monocrystalline with (100) crystalline orientation and 6-degree miscut.

To conduct the etching of the Ge, a custom-made electrochemical cell with the Ge wafer as the working electrode was employed. The electrochemical cell was made of Teflon, with a copper electrode as the backside wafer contact (isolated from the electrolyte) and a platinum counterelectrode. The electrolyte consisted of hydrofluoric acid (49\%) and anhydrous acetic alcohol $(99.9 \%)$ with a ratio of $5: 1(\mathrm{v} / \mathrm{v})$. The current density used was $1.5 \mathrm{~mA} / \mathrm{cm}^{2}$ for the etching and passivation pulses. The duration of pulses was $1 \mathrm{~s}$ for etching (positive current) and $2 \mathrm{~s}$ for passivation (negative current).

Chemical Vapor Infiltration (CVI): To achieve nanographene coating within the MP-Ge, CVI was carried out using a tubular furnace (Carbolite Gero Limited, United Kingdom). In this furnace, the tube and the sample holder boat (MTI corporation, California, USA) are made of quartz. Dihydrogen and acetylene were employed as precursors with argon as the vector gas. The samples were placed vertically during the deposition process. The ratio of acetylene flow to the flow of other gases was $0.89 \%$ (i.e. acetylene flow/dihydrogen + argon flow) and the temperature was controlled with a E1320 controller.

Raman spectroscopy: The Raman measurements were conducted using an AIST-NT OmegaScope system. This system exploits a thermoelectrically cooled Andor CCD detector and a Nanofinder 30 Raman spectrometer. A 0.7 N.A. 100× Mitutoyo MPlan Apo objective was employed to focus a $532.1 \mathrm{~nm}$ laser beam (solid state Cobolt 04-01 series laser source) onto the sample surface.

\section{X-ray Photoelectron Spectroscopy (XPS): X-ray photoelectron spectrometry (XPS)} measurements were performed with a Kratos Axis Ultra XPS DLD. The machine is equipped of an $\mathrm{X}$-ray source of Al monochromatic with an energy of $1486.69 \mathrm{eV}$ with applied power of $225 \mathrm{~W}$. A constant pass energy (PE) mode was used for the analyzer $(\mathrm{PE}=160 \mathrm{eV}$ for the survey scan and Epass $=20 \mathrm{eV}$ for the high-resolution scans). The calibration was done on a metallic Au sample. The binding energy was calibrated for the $4 \mathrm{f}^{7 / 2}$ of metallic Au at $83.96 \mathrm{eV}$. The dispersion of the spectrometer was adjusted to a binding energy of $932.63 \mathrm{eV}$ for the $2 \mathrm{p}^{3 / 2}$ of metallic copper. To compensate the charging effect, the samples were electrically connected to the ground and a charge neutralizer was used. The analyzed area was an oval-shaped spot of $300 \mu \mathrm{m}$ x $700 \mu \mathrm{m}$ surface area. The data was analyzed with the CasaXPS software (version 2.3.18). The experimental Relative Sensitivity Factor (RSF) data as given by Kratos Analytical for their machines applied to quantify 
the XPS spectra. Moreover, a Shirley background was used on all spectra. The charge effect was corrected on the Ge $3 \mathrm{~d}$ peak at $29.3 \mathrm{eV}$. This was done on $\mathrm{Ge}$, but not on carbon.

Transmission Electron Microscopy (TEM): The sample was cleaved in half and the two pieces were glued together with the top surfaces facing each other using epoxy resin. The "sandwich" sample was then glued to a metal puck to be polished. Polishing was done with sandpaper ranging from 400 grit to 4000 grit to reach a mirror-like finish. The sample was polished to attain $100 \mu \mathrm{m}$ $-200 \mu \mathrm{m}$ thickness. Then two $3 \mathrm{~mm}$ disks were cut from it using an ultrasonic disk cutter. The glue line was in the center of the disk. The samples were thinned further down to $80 \mu \mathrm{m}-100 \mu \mathrm{m}$. The samples were then attached to a molybdenum ring to provide support. The samples were dimpled ground to $30 \mu \mathrm{m}$ thickness at the center. Afterwards the samples were ion milled using a Gatan PIPS until a small hole appeared in the center of the samples. The milling parameters were $4.0 \mathrm{kV}$, $4.0 \mathrm{~mA}, 6-7$ degree milling angle, milling time of $4-5$ hours. The microscope is FEI Tecnai at $300 \mathrm{keV}$ and magnification of $410 \mathrm{Kx}$.

Molecular dynamics modeling: Molecular dynamics simulation was performed with Large-Scale Atomic/Molecular Massively Parallel Simulator (LAMMPS) and the results were visualized with Visual Molecular Dynamics (VMD) from University of Illinois at Urbana-Champaign. ${ }^{31-34}$

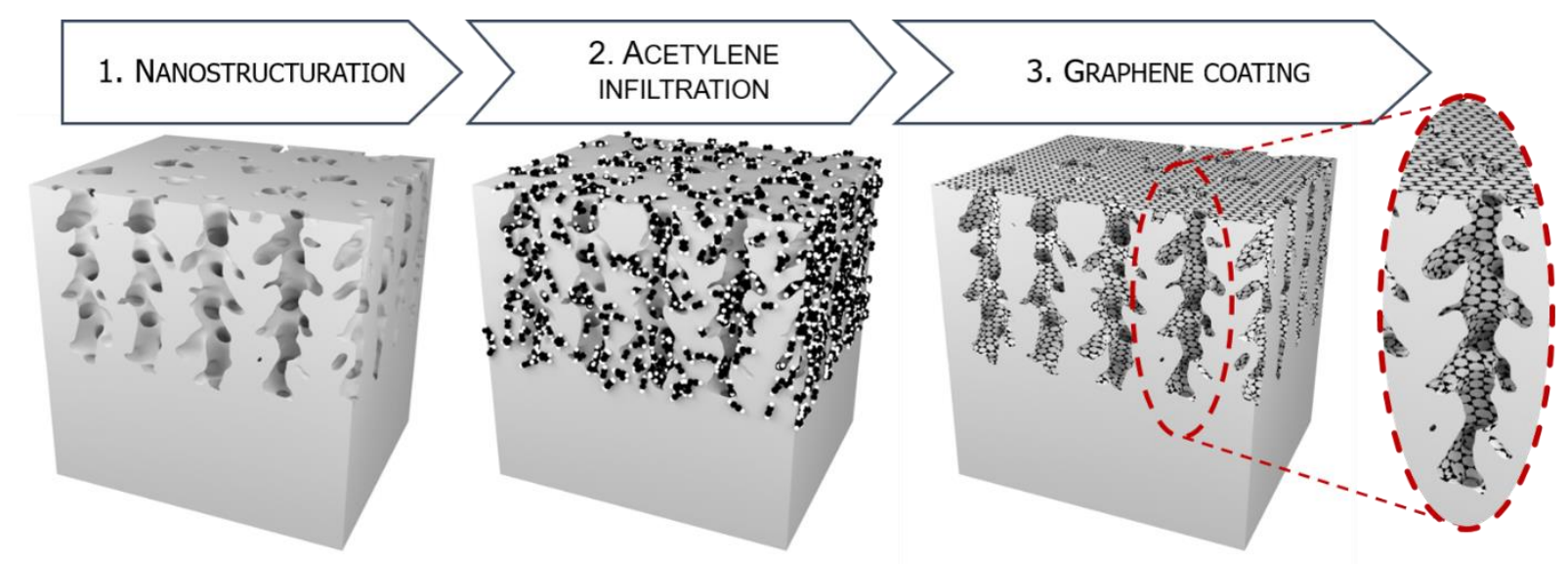

Figure 1: Schematic representation of the three main steps for the synthesis of a Gr-MP-Ge nanomaterial. Step 1. Nanostructuration of (100) germanium substrate using BEE (anodization). Current density was $1.5 \mathrm{~mA} / \mathrm{cm}^{2}$, wafer resistivity was 0.01-0.02 $\Omega . \mathrm{cm}$, electrolyte was made of hydrofluoric acid and ethanol with a ratio of 5:1 (v/v). Step 2. Acetylene infiltration at $110^{\circ} \mathrm{C}$ in a tubular furnace with argon and hydrogen at atmosphere pressure. Step 3. Graphene coating with tubular furnace at carbonization temperature, which was varied in this study.

\section{Results and discussion}

\section{Synthesis of the Gr-MP-Ge: Goal and synthesis steps}


The ultimate material that is expected is a crystalline MP-Ge substrate coated with a thin layer of nanographene, encapsulating all the porous structure. Therefore, the crystalline structure and nanostructuration of fabricated MP-Ge should be maintained during the rest of the process, especially after the carbon coating deposition. Here, the proposed synthesis method consists of three major steps as shown in figure 1. The first step was devised to create the mesoporous structure of Ge using bipolar electrochemical etching (BEE) technique, which can be extended to the full-size wafers for large-scale production of such type of nanomaterial. ${ }^{35}$ It has been demonstrated that the thickness and density of mesoporous layer could be well controlled by the process parameters, such as current density and pulse duration. ${ }^{24-26}$ This step was realized on a (100), single side polished, gallium p-doped Ge wafer with a resistivity of $0.01-0.02 \Omega . \mathrm{cm}$. Electrolyte was made of hydrofluoric acid and ethanol with a ratio of 5:1 (v/v). Then the nanographene coating was carried out through acetylene infiltration in the pores of the Ge substrate, at atmosphere pressure. This step was performed in a tubular furnace with argon and dihydrogen atmosphere at initial temperature of $110{ }^{\circ} \mathrm{C}$. Dihydrogen $\left(\mathrm{H}_{2}\right)$ is a reductant specie and allows to reduce the native oxide present and avoid the formation of new oxide on the sample during the synthesis and the cooling down. Furthermore, presence of $\mathrm{H}_{2}$ in vapor allows to regenerate hydrogenated surface of germanium. ${ }^{36,37}$ Once acetylene was infiltrated in the mesoporous structure, the temperature was raised in order to transform the acetylene molecules into carbon atoms.

Since the carbonization temperature has a direct effect on the quality of the carbon layer, multiple Gr-MP-Ge nanomaterials were synthesized at different carbonization temperature of $400{ }^{\circ} \mathrm{C}-920$ ${ }^{\circ} \mathrm{C}$ (See experimental section for details). ${ }^{37}$

Despite, the two last steps are performed in the same reactor, they represent two critical steps in the synthesis process. First, graphene formation is the direct consequence of the acetylene infiltration which is critical to ensure uniformity in depth of the deposition. Second, temperature treatment is critical to control the quality of the deposition. Therefore, the deposition is highly dependent on the good infiltration but temperature plays also a critical role discussed in the Raman section of this work. 


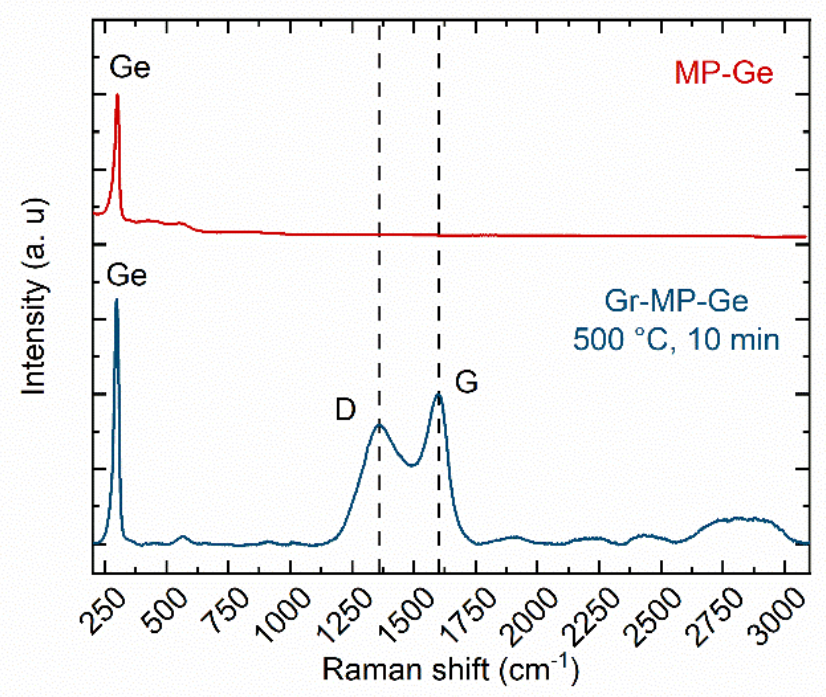

Figure 2 Typical Raman spectra, using $\lambda_{\mathrm{L}}=532 \mathrm{~nm}$, of MP-Ge (red and top line) and Gr-MP-Ge nanomaterial (blue and bottom line) treated at $500{ }^{\circ} \mathrm{C}$ for $10 \mathrm{~min}$, respectively.

\section{Nature, quality and domain size of the carbon coating: Raman spectroscopy}

Figure 2 Typical Raman spectra, using $\lambda \mathrm{L}=532 \mathrm{~nm}$, of MP-Ge (red and top line) and Gr-MP-Ge nanomaterial (blue and bottom line) treated at $500{ }^{\circ} \mathrm{C}$ for $10 \mathrm{~min}$, respectively.

For all samples, treated at $400{ }^{\circ} \mathrm{C}-920{ }^{\circ} \mathrm{C}$, the Raman spectra were collected with a Raman spectrometer using an excitation laser of $532.1 \mathrm{~nm}$ (See figure S1 in the Supporting Information). Figure 2 depicts the typical Raman spectra acquired on MP-Ge (red line) and Gr-MP-Ge nanomaterials (blue line) treated at $500{ }^{\circ} \mathrm{C}$.

The peak observed at $300 \mathrm{~cm}^{-1}$ corresponds to the $\mathrm{T}_{2 \mathrm{~g}}$ Raman mode of the diamond-type for Ge crystal. Besides, the D and G peaks located at $1350 \mathrm{~cm}^{-1}$ and $1588 \mathrm{~cm}^{-1}-1602 \mathrm{~cm}^{-1}$, respectively, can be considered as a signature of carbon. ${ }^{38}$ Normally, the Raman spectrum of disordered graphite contains two peaks, i.e., the $G$ peak around $1580 \mathrm{~cm}^{-1}-1600 \mathrm{~cm}^{-1}$ and the D peak around $1350 \mathrm{~cm}^{-}$ 139 The presence of disorder and defects inside the $\mathrm{sp}^{2}$ film are likely responsible for $\mathrm{D}$ peak. Additionally, the 2D peak region has a low intensity and important broadness for the sample treated at $500^{\circ} \mathrm{C}$. The shape is modified with higher temperature treatment (Figure $\mathrm{S} 1 \mathrm{~b}$ ) with appearance of marked $2 \mathrm{D}$ and $\mathrm{D}+\mathrm{D}$ ' peaks. These changes are due to the decrease of disorder with the increasing temperature. ${ }^{40}$ The $\{\mathrm{I}(\mathrm{D}) / \mathrm{I}(\mathrm{G})\}$ ratio allows to extract information about the nature of the materials such as the domain size within the coating layer. Based on the work of Ferrari and Robertson $^{39}$, the $\{\mathrm{I}(\mathrm{D}) / \mathrm{I}(\mathrm{G})\}$ ratio increased with the carbonization temperature and the $\mathrm{G}$ peak position shifted towards $1600 \mathrm{~cm}^{-1}$ that indicates the transition from graphite towards nano-graphite (stage 1), with the presence of only $\mathrm{sp}^{2}$ hybridization for carbon-carbon bonding. Furthermore, as 
noticed on similar material as mesoporous silicon, the number of layer is estimated at 2-3 layers and the growth is self-limiting catalytic reaction. ${ }^{15,41}$ Considering all these information, we call the carbon coating studied here, nanographene.

In stage 1, the relation of Tuinstra and Koenig is applicable. This relation explains that the ratio of intensities of $\mathrm{D}$ and $\mathrm{G}$ peaks is inversely proportional to the domain size, $\mathrm{L}_{\mathrm{a}}$, as follows: ${ }^{38,39,42}$

$$
\frac{I(D)}{I(G)}=\frac{C(\lambda)}{L_{a}}
$$

where $\mathrm{C}(\lambda=532 \mathrm{~nm})$ is $\sim 49.6 \AA$ according to the equation $\mathrm{C}(\lambda) \approx \mathrm{C}_{0}+\lambda_{\mathrm{L}} \mathrm{C}_{1}$, in which $\mathrm{C}_{0}$ and $\mathrm{C}_{1}$ are estimated to be $-126 \AA$ and 0.033 respectively. ${ }^{43}$

Figure 3 displays $\{\mathrm{I}(\mathrm{D}) / \mathrm{I}(\mathrm{G})\}$ ratio variations as well as the evolution of the domain size $\mathrm{L}_{\mathrm{a}}$, calculated using equation (1), versus the carbonization temperature. As it can be seen, the domain size, $\mathrm{L}_{\mathrm{a}}$, was changing from $\sim 8$ to $3 \mathrm{~nm}$ for samples that were carbon coated at 400 to $920{ }^{\circ} \mathrm{C}$, respectively. Therefore, $\mathrm{L}_{\mathrm{a}}$ decreases with increasing the carbonization temperature suggesting that higher temperature favored creation of more nucleation sites leading to more grain boundaries. The combination of the observations of the $\mathrm{I}(\mathrm{D}) / \mathrm{I}(\mathrm{G})$ and the $2 \mathrm{D}$ peak shape indicates that the domains of graphene are becoming smaller but with a higher crystalline quality with increasing treatment temperature.

The most important deduction is the tunability of the domain size by a factor of 2. Evidently, lower carbonization temperature resulted in lower $\mathrm{I}(\mathrm{D}) / \mathrm{I}(\mathrm{G})$ ratio, which is desired for synthesis of less grain boundary nanographene layer. In contrast, Raman spectra of the sample that was treated at $400{ }^{\circ} \mathrm{C}$ (see Figure S1 in the Supporting Information) attested the presence of carbon and suggested such a temperature is the limitation for the growth and the thermal cracking of the acetylene precursor. Therefore, a carbonization temperature higher will be chosen for the next. Consequently, $500{ }^{\circ} \mathrm{C}$ is very close to the optimal condition for large domain size and maintaining the porosity of the substrate. Furthermore, molecular dynamic modeling will show later in the paper, the good reactivity at this temperature. 


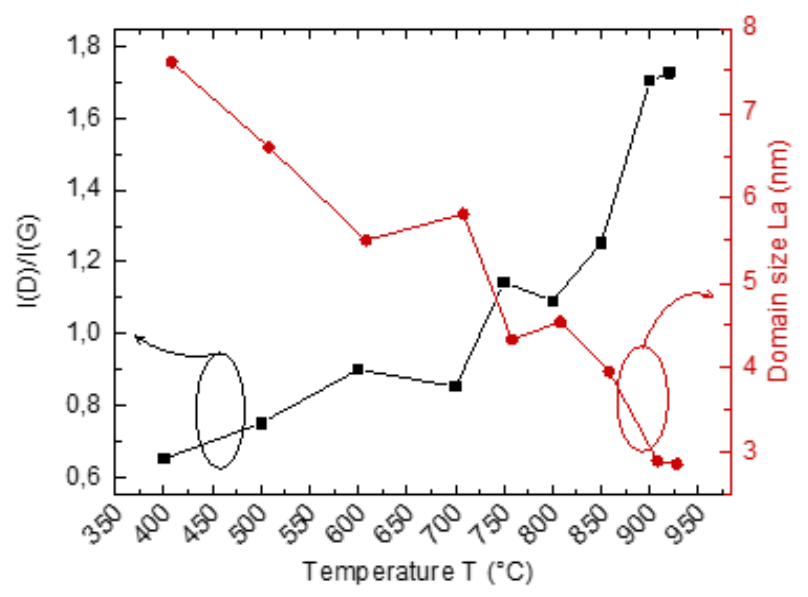

Figure 3 Evolution of the $\mathrm{I}(\mathrm{D}) / \mathrm{I}(\mathrm{G})$ ratio and domain size, La, of graphene coating as a function of carbonization temperature.
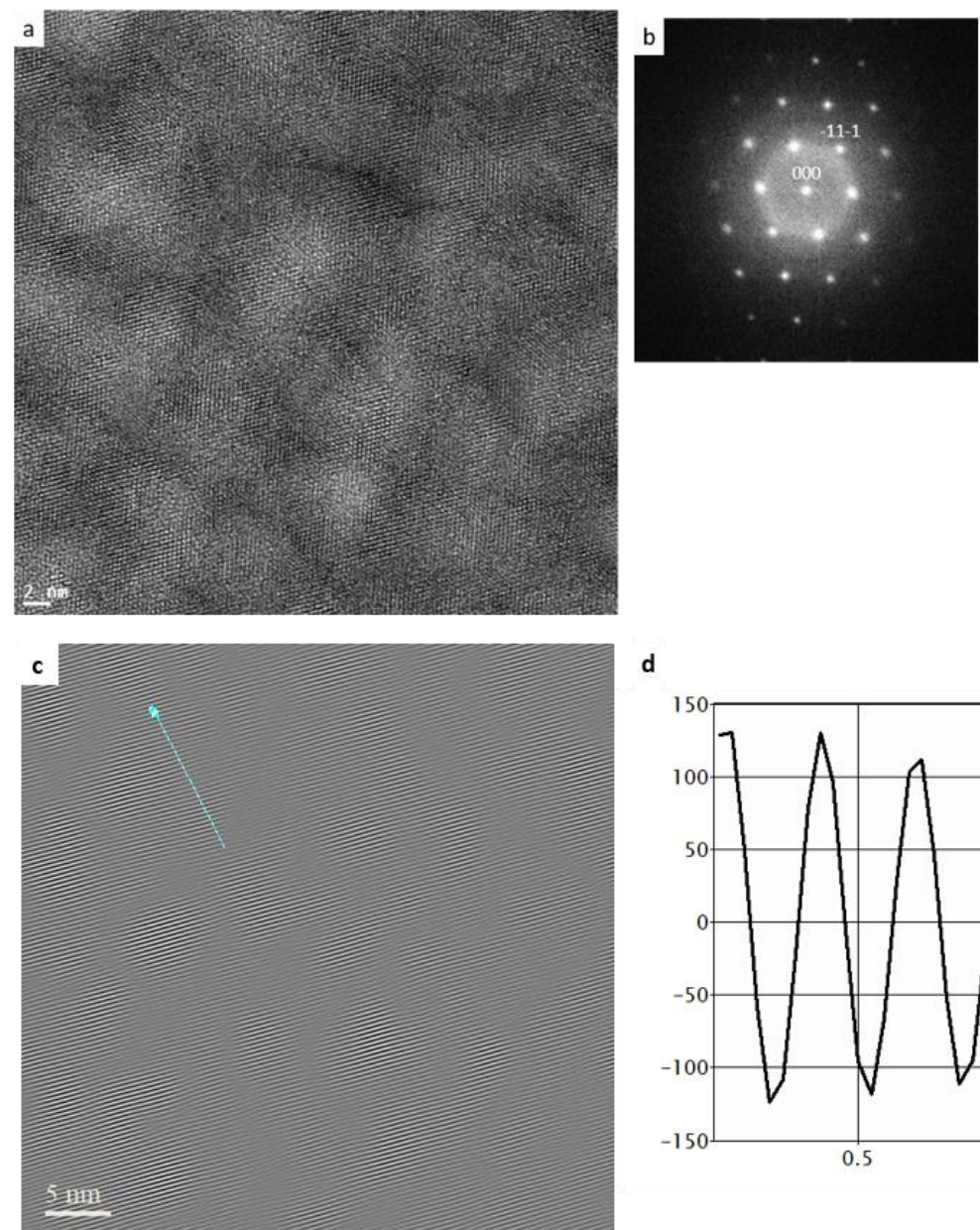

d

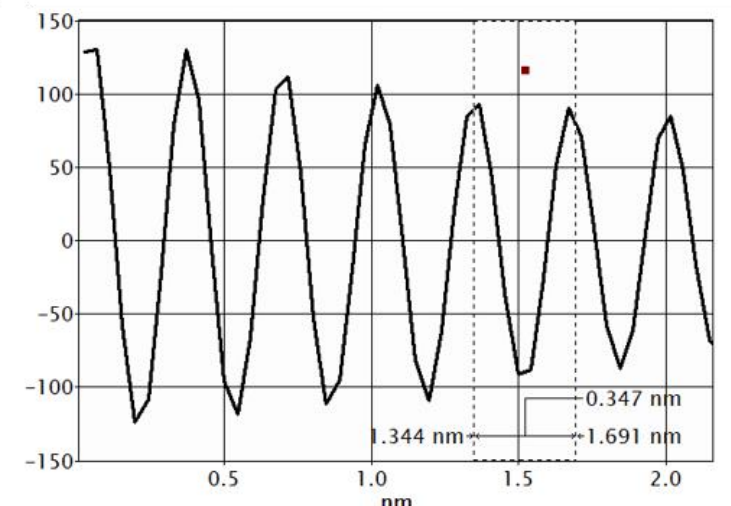

Figure 4 (a) HR-TEM image of a cross-section of Gr-MP-Ge nanomaterial with an electron beam set at $300 \mathrm{keV}$ and magnification of $410 \mathrm{Kx}$, (b) diffraction image obtained by FFT, (c) IFFT image of diffraction image present in (a) using a mask to keep only the spot for (-111) and (1-11), here the solid blue line denotes the physical location of obtained profile (d) Profile of the distance between the planes for $\{111\}$.

\section{MP-Ge with crystalline structure: TEM}


To characterize the crystallinity of the proposed nanomaterial, TEM measurements were carried out with an electron beam at $300 \mathrm{keV}$. For this purpose, only the porous part is observed in crosssection.

Figure 4a presents the High-Resolution TEM (HR-TEM) image exhibiting germanium crystal planes taken from a Gr-MP-Ge synthesized at $500{ }^{\circ} \mathrm{C}$. Figure $4 \mathrm{a}$ is the direct observation using TEM for Gr-MP-Ge sample and allows to extract the diffraction image presenting in figure $4 \mathrm{~b}$. The different shades of gray are related to the difference in the thickness of the sample. The diffraction image in figure $4 \mathrm{~b}$ was obtained by applying Fast Fourier Transform (FFT) on the HR-TEM image, shown in figure $4 \mathrm{a} .{ }^{44}$ As can be seen in figure $4 \mathrm{~b}$, diffraction image contains spots with a welldefined periodic pattern. Each spot represents an atomic plane of Ge and the distance between the central spot, marked with (000) and the spot marked (-11-1), is $3.47 \AA$.

For a cubic crystal, the relation between the lattice constant $\mathrm{a}_{0}$, the Miller indices $(\mathrm{h}, \mathrm{k}, \mathrm{l})$ and the lattice spacing $\mathrm{d}$ is defined based on the Bragg's law as follows:

$$
d=\frac{a_{0}}{\sqrt{h^{2}+k^{2}+l^{2}}}
$$

Based on the equation (2), the lattice constant $\mathrm{a}_{0}$ is found to be $5.9 \AA$ corresponding to the lattice parameter of Ge.

Figure 4c displays the Inverse Fast Fourier Transform (IFFT) of the diffraction image presented in figure 4b. Prior to applying the IFFT, a mask was applied on the image for selecting only the two spots representing (-111) and (1-11) planes. In figure 4c, the planes of Ge corresponding to the lattice plane family of $\{111\}$ can be clearly identified. Figure $4 \mathrm{~d}$ illustrates the profile of pixels in the image shown in figure $4 \mathrm{c}$. Here, intensity of the observed peaks is arbitrary and it depends on the image contrast. However, the distance between these peaks corresponds to the distance between the planes of Ge.

TEM data confirmed that the single-crystallinity of Ge was preserved after porosification and carbon coating. Furthermore, the aim is to keep the high surface area in maintaining the morphology of the porous material after graphene growth which is confirmed with SEM observations (see figure S2). SEM observations highlights a change in the distribution of size of the crystallites due to the temperature treatment (re-densification of the crystallites increasing their size). In spite of that the morphology of the porous structure is keeping mesoporous with a thickness of around $500 \mathrm{~nm}$ and a high specific surface area. It is the aim of our process to preserve the mesoporous structure on a sufficient thickness to have a large specific surface area in comparison with the bulk. Although the 
TEM characterization confirmed the crystalline structure of $\mathrm{Ge}$, the carbon component could not be studied using this technique. In fact, during the sample preparation for TEM imaging, an epoxy made of carbon material was utilized. Consequently, distinguishing the two materials in diffraction images and other measurements was not possible.

TEM measurements and Raman spectroscopy data showed that the crystallinity of Ge was preserved, while the carbon coating resulted in the formation of nanographene with disorder mostly related to the grain boundaries. Then, in order to characterize the bonding nature between MP-Ge and nanographene, XPS measurements were conducted.

\section{Bonding of carbon to MP-Ge: XPS}

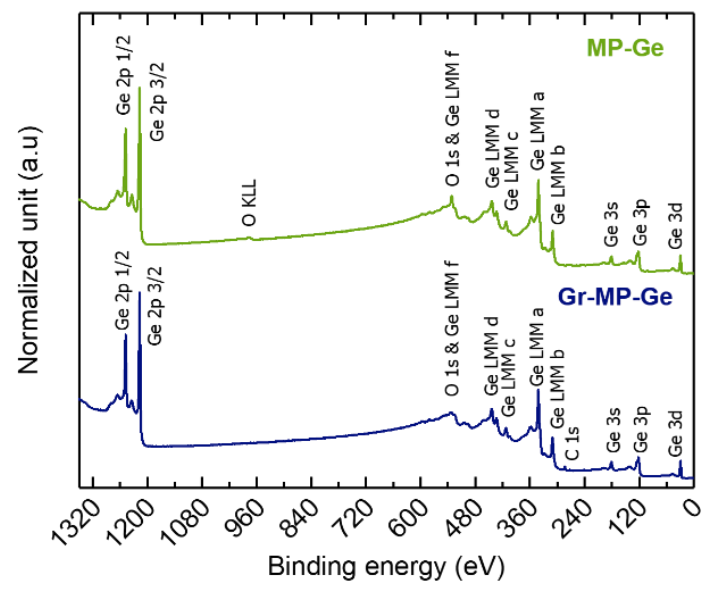

Figure 5 Survey XPS spectra, with an Al monochromatic source $(1486.69 \mathrm{eV})$. Spectra were taken from MP-Ge and Gr-MP-Ge nanomaterial after Ar ions bombardment, to determine which elements were present. Offset on spectra are done in order to enhance the quality of reading.
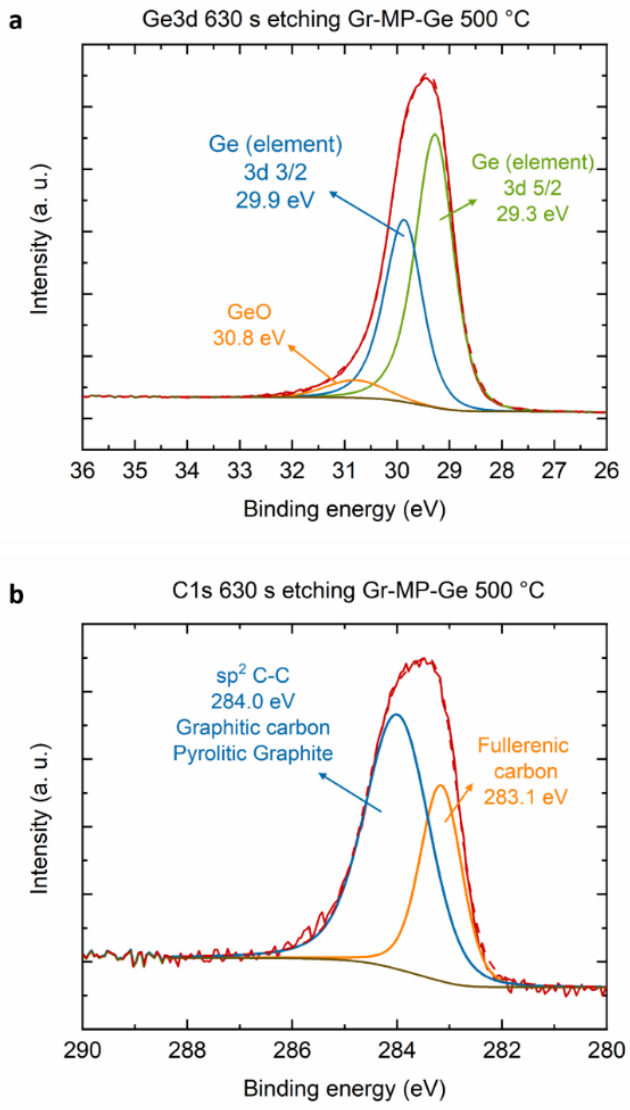

Figure 6 (a) XPS spectra of Ge 3d core energy for GrMP-Ge after Ar ions bombardment, (b) XPS spectra of $\mathrm{C} 1 \mathrm{~s}$ core energy for $\mathrm{Gr}-\mathrm{MP}-\mathrm{Ge}$ after $\mathrm{Ar}$ ions bombardment.

(A color version of this figure can be viewed online.) 
Figure 5 compares the XPS survey spectra obtained from MP-Ge and Gr-MP-Ge nanomaterial. According to these spectra, the main chemical elements are Ge, oxygen and carbon. The MP-Ge spectrum contains two peaks corresponding to the oxygen, O1s at $531.14 \mathrm{eV}$ and OKLL at $976 \mathrm{eV}$. Whereas XPS spectrum of the Gr-MP-Ge nanomaterial has only the O1s peak, which is shifted to $533.00 \mathrm{eV}$; besides, it is less intense and less sharp compared to the XPS spectrum of MP-Ge. This may suggest that the graphene slightly passivated the Ge surface and partially prevented oxide formation. Furthermore, the survey spectra for both samples show the presence of Ge peaks at 1218 $\mathrm{eV}, 1251 \mathrm{eV}, 29.3 \mathrm{eV}$ related to, respectively, Ge2p $\mathrm{p}^{1 / 2}, \mathrm{Ge} 2 \mathrm{p}^{3 / 2}$ and Ge 3d. Moreover, XPS peaks related to $\mathrm{Ge} 3 \mathrm{p}$ and $\mathrm{Ge} 3 \mathrm{~s}$ are presented at $122.2 \mathrm{eV}$ and $181.1 \mathrm{eV}$, respectively. The peak related to carbon $\mathrm{C} 1 \mathrm{~s}$ at $283.2 \mathrm{eV}$ is only present for Gr-MP-Ge. In order to determine the bonding condition between the Ge and carbon, high-resolution XPS scans on these two specific elements were collected.

Figure 6 shows high-resolution XPS scans of the Ge3d at $29.5 \mathrm{eV}$ and C1s at $283.5 \mathrm{eV}$, taken after $630 \mathrm{~s}$ argon ion etching ( $\mathrm{Ar}^{+}$sputtering) of the samples. Argon ions etching allows to remove a part of the surface and investigate material properties located at deeper distance from the surface. It also allows removing the adventitious carbon contaminations from the XPS chamber and at the surface of samples. The Lorentzian asymmetric (LA) function fitting of the Ge3d in high-resolution XPS spectrum gives two peaks corresponding to Ge element. A Gaussian/Lorentzian product lineshape is used with a $30 \%$ Lorentzian component for fitting the germanium oxide at $30.8 \mathrm{eV}$ as shown in figure $6 \mathrm{a}^{45}$ The XPS peaks at binding energies of $29.3 \mathrm{eV}$ and $29.9 \mathrm{eV}$ correspond to Ge elementary $3 \mathrm{~d}^{5 / 2}$ and $3 \mathrm{~d}^{3 / 2}$, respectively. The small peak at $30.8 \mathrm{eV}$ represents the $\sigma$ bonding between $\mathrm{Ge}$ and oxygen $(\mathrm{GeO})$. The intensity of this peak is also very low, which points out toward small quantity of this bonding. Similarly, the Lorentzian asymmetric (LA) function fitting C1s curve provided two peaks, as shown in figure $6 \mathrm{~b}$. The first peak is located at $284.0 \mathrm{eV}$ and it represents the $\mathrm{sp}^{2}$ carboncarbon bonding. The second peak is located at $283.1 \mathrm{eV}$ and corresponds to fullerenic carbon. ${ }^{46,47}$ Grieco et al. suggested that the $\mathrm{sp}^{2}$-bonded carbon atoms in pentagonal and hexagonal rings give a cage-like polyhedral geometry to fullerenic carbon. ${ }^{48}$. The intensity of this peak linearly increased with the etching time (Figure S3) indicating that the ion bombardment might induce defects. ${ }^{47}$ Indeed, in order to investigate the uniformity of the deposition in depth into the porous structure, XPS high-resolution spectra for Ge3d and C1s are taken at different time of etching with Ar ions bombardment (see figure S3). Before etching, C1s presents only one peak at $284.1 \mathrm{eV}$, after 30 s of etching, two peaks are present, the first one at $284.2 \mathrm{eV}$ and the second at $283.1 \mathrm{eV}$. This second peak appears only after etching and its intensity linearly increases with the time of etching. We 
conclude that this peaks is related to defects created during the bombardment for the etching of the surface. At $6640 \mathrm{~s}$ of etching, carbon is no longer present and the Ge3d peak is perfectly similar to the one obtained on germanium monocrystalline substrate. It is assumed that the entire porous layer was etched and the germanium substrate is reached. As a result of this, carbon is present in all the depth of the porous layer. It is worth noting that no peak related to $\mathrm{sp}^{3}$ bonding and Ge-C bonding were observed. Thus, the XPS data indicate that there is no covalent bond between Ge and carbon. In fact, the dominant bonds are $\mathrm{Ge}-\mathrm{Ge}$ as well as $\mathrm{sp}^{2} \mathrm{C}-\mathrm{C}$.

\section{Molecular Dynamics Modeling}

To further study the mechanism of carbon coating growth, molecular dynamics simulations were conducted. ${ }^{49,50}$ In this modeling the initial substrate was a crystalline bulk Ge with (100) orientation rather than MP-Ge substrate. Despite morphological differences with the mesoporous structure, the chemical interaction between the Ge and carbon remains unchanged. As a first attempt, all the precursors were carbon atoms (Figure S4). In the next step, two kind of calculations are brought off, first with acetylene molecules $\left(\mathrm{C}_{2} \mathrm{H}_{2}\right)$ and the second one with $\mathrm{C}_{2} \mathrm{H}$ radicals, in order to study conditions closer to those of real experimental conditions and to identify the decomposition leading to the deposition. Temperature variations were studied in order to determine the optimal conditions for improving quality of the growing graphene as well as efficient temperature for cracking of acetylene. Therefore, various temperatures, close to those of the experimental conditions, were simulated; specifically, 200, 500, 700, $900{ }^{\circ} \mathrm{C}$. The Ge-Ge and Ge-C interactions were described based on the Tersoff potential. In addition, the Tersoff mixing rules were used for Ge-C interactions as a first attempt. ${ }^{51}$ Then, Ge-H interactions were added based on the non-bonded interactions of Universal Force Field (UFF) leading to the Lennard-Jones potential $V_{G e-H}(r)$, as follows: ${ }^{52}$

$$
V_{G e-H}(r)=4 \epsilon\left[\left(\frac{\sigma}{r}\right)^{6}-\left(\frac{\sigma}{r}\right)^{12}\right]
$$

where $\sigma=3.62 \AA$ and $\epsilon=0.0546 \mathrm{eV}$. Besides, C-C and $\mathrm{C}-\mathrm{H}$ interactions were described using Reactive Empirical Bond-Order (REBO) potential for $\mathrm{C}_{2} \mathrm{H}$ and $\mathrm{C}_{2} \mathrm{H}_{2}$ precursors of deposition simulations. ${ }^{53}$

Three runs of molecular dynamics simulations were carried out with, respectively, 1000 carbon atoms, $500 \mathrm{C}_{2} \mathrm{H}_{2}$ molecules, and $500 \mathrm{C}_{2} \mathrm{H}$ radicals. Molecules were introduced one after each other towards the Ge (100) periodic slab (5 $\mathrm{a}_{0} \times 5 \mathrm{a}_{0} \times 3 \mathrm{a}_{0}, \mathrm{a}_{0}=5.66 \AA$ ) every 2000-time step (dt). Hence, the same number of carbon atoms was released toward the surface. For $\mathrm{C}$ deposition, $\mathrm{dt}=1 \mathrm{fs}$, 
whereas for $\mathrm{C}_{2} \mathrm{H}$ and $\mathrm{C}_{2} \mathrm{H}_{2} \mathrm{dt}=0.1 \mathrm{fs}$, considering fast motion of the carbon bonded hydrogen. During molecular modeling, a Langevin thermostat maintained temperature of vapor and Ge substrate, for mimicking equilibrium thermal CVI. ${ }^{54}$

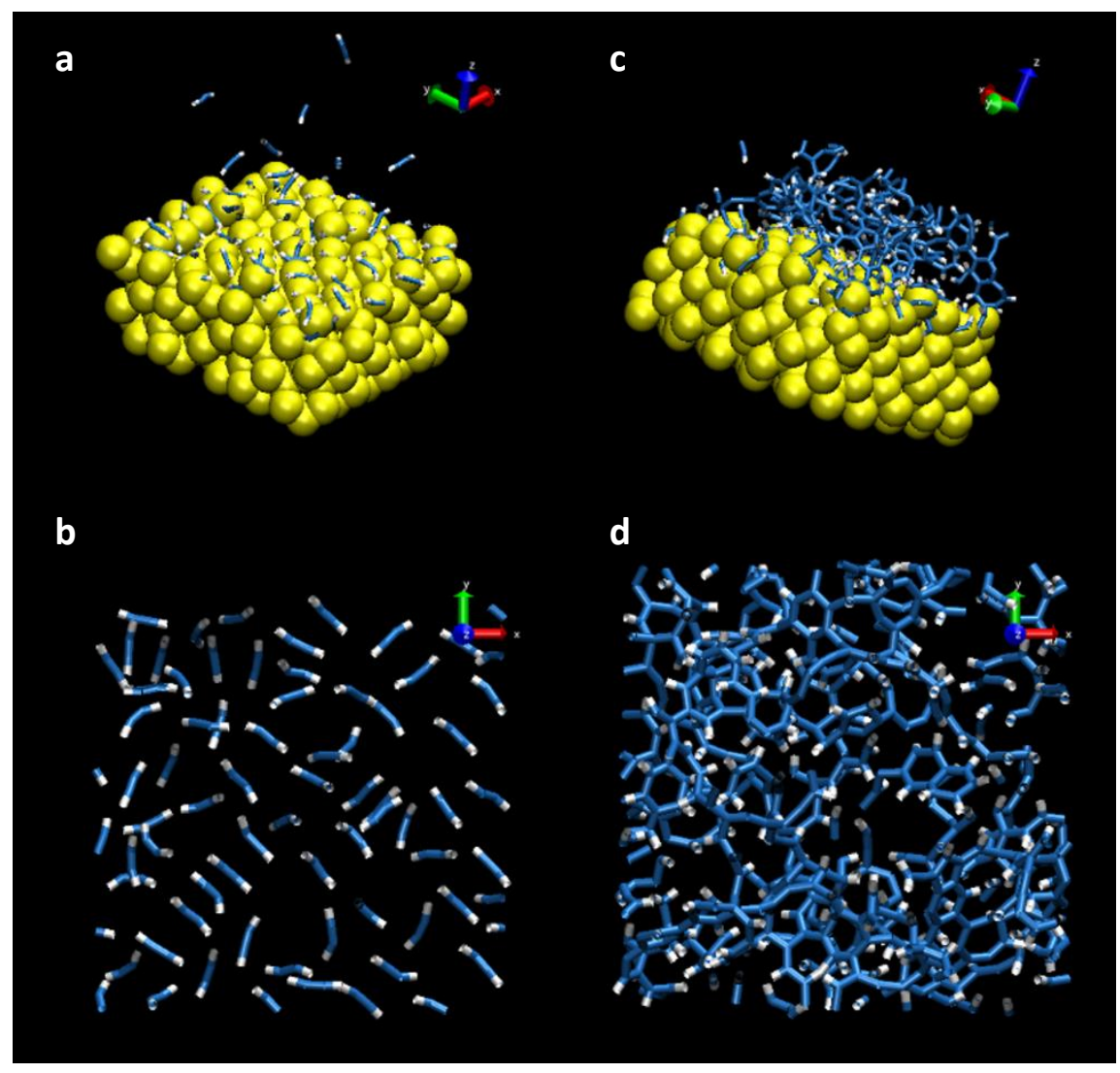

Figure 7 Profile view (a) and top view (b) obtained with the molecular modeling using Tersoff potentials of growth with only acetylene molecules as carbon precursors at $500{ }^{\circ} \mathrm{C}$, Profile view (c) and top view (d) of Graphene growth at $500{ }^{\circ} \mathrm{C}$ with addition of $\mathrm{C}_{2} \mathrm{H}$ radicals as carbon precursors.

Germanium substrate is not represented for snapshots (d) and (f) for further clarity; thus, only carbon-carbon and hydrogen-carbon bonds are represented.

(A color version of this figure can be viewed online, yellow spheres are $\mathrm{Ge}$ atoms, blue ticks are $\mathrm{C}-\mathrm{C}$ bonds and blue-white ticks are $\mathrm{C}-\mathrm{H}$ bonds)

Figure 7 shows the snapshots of the simulation at $500{ }^{\circ} \mathrm{C}$, for two precursors, which were $500 \mathrm{C}_{2} \mathrm{H}_{2}$ molecules and then $500 \mathrm{C}_{2} \mathrm{H}$ radicals. Figure $7 \mathrm{a}$ and $7 \mathrm{~b}$ present results of the molecular modeling at $500{ }^{\circ} \mathrm{C}$ with acetylene molecules as precursors. Figure $7 \mathrm{c}$ and $7 \mathrm{~d}$ represent the results for the case in which $\mathrm{C}_{2} \mathrm{H}$ radicals were used as precursors, at $500{ }^{\circ} \mathrm{C}$. Since we were interested in bond formation of $\mathrm{C}$ on Ge surface, the snapshots only show the atomic arrangements of Ge atoms and all the covalent bonds of carbon atoms. The covalent bonds in blue are between carbon atoms (CC) and white ones are between carbon and hydrogen atoms $(\mathrm{C}-\mathrm{H})$. The $\mathrm{C}-\mathrm{C}$ and $\mathrm{C}-\mathrm{H}$ bonds are represented without the atoms, for clearly highlighting carbon ring domains. 
Based on figure 7c and 7d, clearly, $\mathrm{C}_{2} \mathrm{H}$ deposition led to growth, while $\mathrm{C}_{2} \mathrm{H}_{2}$ molecules did not react, as can be seen in figure $7 \mathrm{a}$ and $7 \mathrm{~b}$. Moreover, figure $7 \mathrm{c}$ and $7 \mathrm{~d}$ present organized rings carbon which are pentagon and hexagon and rarely heptagon. Finally, in the case of acetylene molecules as precursors and growth temperature of $500{ }^{\circ} \mathrm{C}$, molecules are adsorbed without reactivity on the surface. Nevertheless, consistent with XPS data, no covalent bonds between the Ge and the carbon atoms, has been observed.

In the case of the $\mathrm{C}_{2} \mathrm{H}$ vapor, graphene domains are formed indicating that the presence of radical is required for nucleation and growth of the graphenic coating. Even if it is not perfectly flat and organized, the deposition is not amorphous carbon as Raman spectroscopy and XPS measurements previously depicted attested. Amorphous carbon corresponds to stage 2 in ref. 39 with a downshift of the $\mathrm{G}$ peak and a proportion of almost $20 \%$ of $^{\mathrm{sp}^{3}}$ hybridization. ${ }^{39}$

In order to evaluate the organization of the deposition, Radical Pair Distribution Function $(\mathrm{g}(\mathrm{r}))$ (RPDF) is used to analysis calculations done at different temperature and with $\mathrm{C}_{2} \mathrm{H}$ radical precursors. First, a perfect layer of graphene obtained with modeling dynamic modeling is taken as reference and RPDF is applied. For graphene, the number of first neighbor is 3 atoms and that of second neighbor is 9 atoms. The distance between one atom of carbon and the first neighbor is $1.425 \AA$ and $2.475 \AA$ for the second one. The same analysis, achieved on the simulations at 200 , 500, 700 and $900{ }^{\circ} \mathrm{C}$ with $\mathrm{C}_{2} \mathrm{H}$ precursors, gave the same distances between the close neighbor. It means that coating shows a structure close from the graphene one. The number of neighbor is also pointed out. In average, the number of first neighbor is 2.5 atoms which attested the presence of defects in the form of pentagon, aliphatic chain or incomplete ring.

The molecular dynamic modeling, in good agreement with the experimental findings, elucidated some underlying mechanisms responsible for growth of nanographene on MP-Ge substrate during the CVI process. First, it showed that there was no diffusion of carbon atoms inside Ge substrate. Second, evidently, acetylene molecules alone cannot react directly with the Ge surface, even at high temperature and growth of graphene on Ge surface (100) requires the existence of radicals. This means radicals, such as $\mathrm{C}_{2} \mathrm{H}$, must appeared. Calculations performed with $\mathrm{C}_{2} \mathrm{H}_{2}$ and $\mathrm{H}_{2}$ at $500{ }^{\circ} \mathrm{C}$ exhibited radicals in vapor phase far from the surface attesting the formation in the vapor phase (see figure S6). However, a competitive reaction involving Ge radicals, as suggested by Share et al. in the case of silicon, cannot be discarded. ${ }^{15}$ Moreover, the further decomposition of $\mathrm{C}_{2} \mathrm{H}$ radicals occurring at the surface of Ge certainly involved such mechanism. Germanium radicals on the top of the surface could participate in this decomposition and form carbon atoms which reorganise through thermally stimulated processes, to form the graphene film. ${ }^{37}$ Germanium surface 
reorganizes it in dimers to reduce the number of dangling bonds. This reorganization impacts drastically the reactivity of the surface toward gas-phase reactants. ${ }^{17,55}$ Our assumption is that the carbon precursors are decomposed in the vapor phase and then are captured by the vacancies of germanium dimers before reorganize them through thermally stimulation processes to form graphene film. Third, as was shown with Raman spectroscopy data, modeling outcomes confirmed formation of nanographene structure, while in good correlation with XPS data, modeling indicated that $\mathrm{C}-\mathrm{Ge}$ covalent bonding was not created.

\section{Conclusions}

We developed a new Graphene Mesoporous-Germanium nanomaterial that integrates directly graphene into the mesoporous structure. As a result, 2D graphene became 3D graphene by conforming to the porous structure. First, a study of the D and G peaks that appeared in the Raman spectra indicates the temperature dependency of the nanographene coating quality. Decreasing carbonization temperature improved the domain size of nanographene signifying less grain boundaries. Moreover, the nanographene coating is composed of $\mathrm{sp}^{2} \mathrm{C}-\mathrm{C}$ covalent bonding and did not have any observable Ge-C covalent bonding demonstrating that the graphene integrity and related properties are preserved. Therefore, the domain size of the carbon coating is tunable with the carbonization temperature, which allows adjusting properties such as electrical and thermal conductivity.

The synthesis mechanism at work was investigated by molecular dynamics modeling showing that only $\mathrm{C}_{2} \mathrm{H}$ radicals react with Ge surface and that acetylene molecules could not directly react with Ge. In an excellent agreement with experimental data, modeling data showed that graphene film could be obtained at $500{ }^{\circ} \mathrm{C}$ with radicals, and, no covalent bonds would be formed between Ge and $\mathrm{C}$.

Finally, mesoporous Ge shows a high degree of crystallinity even after all the process.

The successful synthesis of this nanostructured material opens opportunities in a broad range of applications such as epitaxial growth of material, energy storage systems, health, thermoelectric devices or microelectronics., ${ }^{1,56}$ Indeed, in order to combine the respective advantages of semiconductors and graphene, hybrid or composite materials were developed with the aim of directly applying it to a Complementary Metal-Oxide-Semiconductor (CMOS) based manufacturing process. ${ }^{3,57}$ Therefore, direct growth of graphene on semiconductors needs to be developed with the objective of integrating the method within the CMOS industry, which is incompatible with the use of metal. ${ }^{57}$ Recently, some important breakthrough in this area has been 
achieved. ${ }^{37,58}$ These achievements offer the opportunity to incorporate the remarkable properties of graphene for notable enhancement of semiconductor-based devices, possibly beyond the limits set by their nature or fabrication processes. As a matter of fact, the next decades will be dedicated to the introduction of graphene in semiconductor fabrication lines for direct integration on devices. ${ }^{3,57}$ The limitation of 2D graphene is exceeded with the incorporation of graphene in $3 \mathrm{D} .{ }^{59,60} \mathrm{CMOS}$ are not the only application possible, possible applications for this kind of material are wide and numerous and could be analogous to similar materials, such as the graphene-mesoporous silicon, with demonstrated applications like compliant substrates for heteroepitaxy, supercapacitors, drug delivery, sensor applications. ${ }^{61-63}$

\section{Conflicts of interest}

There are no conflicts to declare.

\section{Acknowledgements}

This research was supported by the Natural Sciences and Engineering Research Council of Canada (NSERC), within the context of Discovery grant Strategic Partnership grants, by Quebec Centre for Advanced Materials (QCAM), InnovÉÉ and Fonds de Recherche Nature et Technologies from Quebec government (FRQNT).

The authors thank all the funders. The authors thank Sonia Blais for all the support on XPS measurement and analysis, Guillaume Beaudoin for assistance on Blender software, Guillaume Bertrand for technical help, Arthur Dupuy for fruitful discussions, and all the technical staff of the 3IT for providing technical assistance to the project. Gitanjali Kolhatkar is thankful for a postdoctoral fellowship from the Alexander von Humboldt Foundation.

\section{Footnotes}

Electronic Supplementary Information (ESI) is available online or from the author. Raman spectrum of mesoporous germanium (MP-Ge), as the reference sample, in comparison with the spectra of Graphene-Mesoporous Germanium (Gr-MP-Ge) samples treated at different carbonization temperatures. 


\section{References}

1 A. C. Ferrari, F. Bonaccorso, V. Fal'ko, K. S. Novoselov, S. Roche, P. Bøggild, S. Borini, F. H. L. Koppens, V. Palermo, N. Pugno, J. A. Garrido, R. Sordan, A. Bianco, L. Ballerini, M. Prato, E. Lidorikis, J. Kivioja, C. Marinelli, T. Ryhänen, A. Morpurgo, J. N. Coleman, V. Nicolosi, L. Colombo, A. Fert, M. Garcia-Hernandez, A. Bachtold, G. F. Schneider, F. Guinea, C. Dekker, M. Barbone, Z. Sun, C. Galiotis, A. N. Grigorenko, G. Konstantatos, A. Kis, M. Katsnelson, L. Vandersypen, A. Loiseau, V. Morandi, D. Neumaier, E. Treossi, V. Pellegrini, M. Polini, A. Tredicucci, G. M. Williams, B. H. Hong, J.-H. Ahn, J. M. Kim, H. Zirath, B. J. van Wees, H. van der Zant, L. Occhipinti, A. D. Matteo, I. A. Kinloch, T. Seyller, E. Quesnel, X. Feng, K. Teo, N. Rupesinghe, P. Hakonen, S. R. T. Neil, Q. Tannock, T. Löfwander and J. Kinaret, Nanoscale, 2015, 7, 4598-4810.

2 K. S. Novoselov, A. K. Geim, S. V. Morozov, D. Jiang, Y. Zhang, S. V. Dubonos, I. V. Grigorieva and A. A. Firsov, Science, 2004, 306, 666-669.

3 W. Kong, H. Kum, S.-H. Bae, J. Shim, H. Kim, L. Kong, Y. Meng, K. Wang, C. Kim and J. Kim, Nat. Nanotechnol., 2019, 14, 927-938.

4 S. Zhu, Y. Song, X. Zhao, J. Shao, J. Zhang and B. Yang, Nano Res., 2015, 8, 355-381.

5 M. Bacon, S. J. Bradley and T. Nann, Particle \& Particle Systems Characterization, 2014, 31, 415428.

6 J. Shen, Y. Zhu, X. Yang and C. Li, Chem. Commun., 2012, 48, 3686-3699.

7 S. Zhu, J. Zhang, C. Qiao, S. Tang, Y. Li, W. Yuan, B. Li, L. Tian, F. Liu, R. Hu, H. Gao, H. Wei, H. Zhang, H. Sun and B. Yang, Chemical Communications, 2011, 47, 6858-6860.

8 K. T. Kim, J. W. Jung and W. H. Jo, Carbon, 2013, 63, 202-209.

9 Q. Ke and J. Wang, Journal of Materiomics, 2016, 2, 37-54.

10 A. Jayakumar, V. K. Jose and J.-M. Lee, Small Methods, 2020, 4, 1900735.

11 H. Föll, J. Carstensen and S. Frey, Journal of Nanomaterials, 2006, 2006, e91635.

12 H. Föll, S. Langa, J. Carstensen, M. Christophersen and I. M. Tiginyanu, Advanced Materials, 2003, 15, 183-198.

13 D. Nadarassan, in Handbook of Porous Silicon, ed. L. Canham, Springer International Publishing, Cham, 2018, pp. 927-944.

14 D. G. Papageorgiou, I. A. Kinloch and R. J. Young, Progress in Materials Science, 2017, 90, 75127.

15 K. Share, R. E. Carter, P. Nikolaev, D. Hooper, L. Oakes, A. P. Cohn, R. Rao, A. A. Puretzky, D. B. Geohegan, B. Maruyama and C. L. Pint, J. Phys. Chem. C, 2016, 7. 
16 C. Fang, H. Föll and J. Carstensen, Journal of Electroanalytical Chemistry, 2006, 589, 259-288.

17 P. W. Loscutoff and S. F. Bent, Annual Review of Physical Chemistry, 2006, 57, 467-495.

18 J.-H. Lee, S.-G. Kang, H.-S. Jang, J.-Y. Moon and D. Whang, Advanced Materials, 2019, 31, 1803469.

19R. I. Scace and G. A. Slack, J. Chem. Phys., 1959, 30, 1551-1555.

20 W. Bao, F. Miao, Z. Chen, H. Zhang, W. Jang, C. Dames and C. N. Lau, Nature Nanotech, 2009, 4, 562-566.

21 G. Korotcenkov and B. K. Cho, Critical Reviews in Solid State and Materials Sciences, 2010, 35, $153-260$.

22 H. C. Choi and J. M. Buriak, Chem. Commun., 2000, 1669-1670.

23 E. Garralaga Rojas, B. Terheiden, H. Plagwitz, J. Hensen, C. Baur, G. F. X. Strobl and R. Brendel, Electrochemistry Communications, 2010, 12, 231-233.

24 S. Tutashkonko, A. Boucherif, T. Nychyporuk, A. Kaminski-Cachopo, R. Arès, M. Lemiti and V. Aimez, Electrochimica Acta, 2013, 88, 256-262.

25 Y. A. Bioud, A. Boucherif, A. Belarouci, E. Paradis, S. Fafard, V. Aimez, D. Drouin and R. Arès, Electrochimica Acta, 2017, 232, 422-430.

26 S. Tutashkonko, S. Alekseev and T. Nychyporuk, Electrochimica Acta, 2015, 180, 545-554.

27 X. Li, W. Cai, J. An, S. Kim, J. Nah, D. Yang, R. Piner, A. Velamakanni, I. Jung, E. Tutuc, S. K. Banerjee, L. Colombo and R. S. Ruoff, Science, 2009, 324, 1312-1314.

28 A. Reina, X. Jia, J. Ho, D. Nezich, H. Son, V. Bulovic, M. S. Dresselhaus and J. Kong, Nano Lett., $2009,9,30-35$.

29 K. S. Kim, Y. Zhao, H. Jang, S. Y. Lee, J. M. Kim, K. S. Kim, J.-H. Ahn, P. Kim, J.-Y. Choi and B. H. Hong, Nature, 2009, 457, 706-710.

30 S. Bae, H. Kim, Y. Lee, X. Xu, J.-S. Park, Y. Zheng, J. Balakrishnan, T. Lei, H. Ri Kim, Y. I. Song, Y.-J. Kim, K. S. Kim, B. Özyilmaz, J.-H. Ahn, B. H. Hong and S. Iijima, Nature Nanotechnology, 2010, 5, 574-578.

31 S. Plimpton, Journal of Computational Physics, 1995, 117, 1-19.

32 LAMMPS, http://lammps.sandia.gov.

33 W. Humphrey, A. Dalke and K. Schulten, Journal of Molecular Graphics, 1996, 14, 33-38.

34 VMD, http://www.ks.uiuc.edu/Research/vmd/.

35 A. Loni, in Handbook of Porous Silicon, ed. L. Canham, Springer International Publishing, Cham, 2014, pp. 11-22. 
36 Y. Jin, B. Hu, Z. Wei, Z. Luo, D. Wei, Y. Xi, Y. Zhang and Y. Liu, J. Mater. Chem. A, 2014, 2, $16208-16216$.

37 J.-H. Lee, E. K. Lee, W.-J. Joo, Y. Jang, B.-S. Kim, J. Y. Lim, S.-H. Choi, S. J. Ahn, J. R. Ahn, M.-H. Park, C.-W. Yang, B. L. Choi, S.-W. Hwang and D. Whang, Science, 2014, 344, 286-289.

38 A. C. Ferrari, J. C. Meyer, V. Scardaci, C. Casiraghi, M. Lazzeri, F. Mauri, S. Piscanec, D. Jiang, K. S. Novoselov, S. Roth and A. K. Geim, Phys. Rev. Lett., 2006, 97, 187401.

39 A. C. Ferrari and J. Robertson, Phys. Rev. B, 2000, 61, 14095-14107.

40 A. Merlen, J. Buijnsters and C. Pardanaud, Coatings, 2017, 7, 153.

41 G. Kolhatkar, A. Boucherif, A. Boucherif, A. Dupuy, L. G. Frechette, R. Ares and A. Ruediger, Nanotechnology, , DOI:10.1088/1361-6528/aaac40.

42 F. Tuinstra and J. L. Koenig, J. Chem. Phys., 1970, 53, 1126-1130.

43 M. J. Matthews, M. A. Pimenta, G. Dresselhaus, M. S. Dresselhaus and M. Endo, Phys. Rev. B, 1999, 59, R6585-R6588.

44 D. R. G. Mitchell, Ultramicroscopy, 2008, 108, 367-374.

45 K. T. Wong, Y.-G. Kim, M. P. Soriaga, B. S. Brunschwig and N. S. Lewis, J. Am. Chem. Soc., 2015, 137, 9006-9014.

46 W. Cheng, L.-T. Weng, Y. Li, A. Lau, C. K. Chan and C.-M. Chan, Aerosol Science and Technology, 2013, 47, 1118-1124.

47 W. Xie, L.-T. Weng, K. M. Ng, C. K. Chan and C.-M. Chan, Carbon, 2017, 112, 192-200.

48 W. J. Grieco, J. B. Howard, L. C. Rainey and J. B. Vander Sande, Carbon, 2000, 38, 597-614.

49E. C. Neyts and P. Brault, Plasma Processes and Polymers, 2017, 14, 1600145.

50 L. Schwaederlé, P. Brault, C. Rond and A. Gicquel, Plasma Processes and Polymers, 2015, 12, 764-770.

51 J. Tersoff, Phys. Rev. B, 1989, 39, 5566-5568.

52 A. K. Rappe, C. J. Casewit, K. S. Colwell, W. A. Goddard and W. M. Skiff, J. Am. Chem. Soc., 1992, 114, 10024-10035.

53 D. W. Brenner, O. A. Shenderova, J. A. Harrison, S. J. Stuart, B. Ni and S. B. Sinnott, J. Phys.: Condens. Matter, 2002, 14, 783-802.

54 R. L. Davidchack, R. Handel and M. V. Tretyakov, J. Chem. Phys., 2009, 130, 234101.

55 J. Dabrowski, G. Lippert, J. Avila, J. Baringhaus, I. Colambo, Y. S. Dedkov, F. Herziger, G. Lupina, J. Maultzsch, T. Schaffus, T. Schroeder, M. Kot, C. Tegenkamp, D. Vignaud and M.-C. Asensio, Sci Rep, 2016, 6, 31639.

56 W. Jie and J. Hao, Nanoscale, 2014, 6, 6346-6362. 
57 D. Neumaier, S. Pindl and M. C. Lemme, Nature Materials, 2019, 18, 5.

58 H. Yang, J. Heo, S. Park, H. J. Song, D. H. Seo, K.-E. Byun, P. Kim, I. Yoo, H.-J. Chung and K. Kim, Science, 2012, 336, 1140-1143.

59 P. Samorì, I. A. Kinloch, X. Feng and V. Palermo, 2D Mater., 2015, 2, 030205.

60 J. Biener, S. Dasgupta, L. Shao, D. Wang, M. A. Worsley, A. Wittstock, J. R. I. Lee, M. M. Biener, C. A. Orme, S. O. Kucheyev, B. C. Wood, T. M. Willey, A. V. Hamza, J. Weissmüller, H. Hahn and T. F. Baumann, Advanced Materials, 2012, 24, 5083-5087.

61 Boucherif Abderrahim Rahim, Boucherif Abderraouf, Kolhatkar Gitanjali, Ruediger Andreas and Arès Richard, Small, 2017, 13, 1603269.

62 L. Oakes, A. Westover, J. W. Mares, S. Chatterjee, W. R. Erwin, R. Bardhan, S. M. Weiss and C. L. Pint, Scientific Reports, 2013, 3, 3020.

63 J. Salonen and E. Mäkilä, Advanced Materials, 2018, 30, 1703819. 